\title{
Practical designs for out-of-band emission suppression and adjacent channel interference rejection for orthogonal frequency division multiplexing-based cognitive radios
}

\author{
Juan Fang ${ }^{*}$ and I-Tai Lu
}

\begin{abstract}
Opposite to the conventional wisdom, practical considerations for out-of-band emission (OOBE) suppression and adjacent channel interference $(\mathrm{ACl})$ rejection in orthogonal frequency division multiplexing (OFDM) -based cognitive radio systems are not completely alike. For example, $\mathrm{ACl}$ will increase after discarding the cyclic prefix (CP) because the interference signal is also truncated during the CP removal process. Furthermore, effective OOBE is much larger than the estimated OOBE if there is a timing offset (TO) between the primary and secondary users. To the best of our knowledge, these and some other practical issues have not been well addressed. Various important practical issues (including multipath delay spread, high peak-to-average power ratio (PAPR), spectral efficiency loss, high complexity, sensitivities to frequency and timing offsets, non-contiguous and dynamically changing spectrum, CP removal, spectral containment, etc.) concerning OOBE suppression and $\mathrm{ACl}$ rejection are studied in this paper. Numerical results show that none of the existing approaches can deal with all practical issues satisfactorily. Hybrid combinations of frequency-domain approaches (spectral precoding and PAPR precoding) and time-domain approaches (windowing and filtering) are developed to maintain the merits and mitigate the drawbacks of each constituent approach in order to deal with these practical challenges. It is shown that a combination of filtering and precoding provides the best OOBE suppression and a combination of windowing and precoding provides the best ACl rejection characteristics in our numerical examples.
\end{abstract}

Keywords: OOBE; ACl; Cognitive radio; OFDM

\section{Introduction}

Cognitive radio (CR), as a promising solution to the spectrum congestion problem brought by the rapid advance of wireless communication techniques and the drastic increase of wireless devices, has drawn a lot of attentions recently (e.g., see [1-3]). In CR systems, the secondary users (SUs) are allowed to dynamically access to the spectrum resources, which are not occupied by the primary users (PUs) and could be non-contiguous, without causing interference to the PUs or other SUs transmitted over adjacent channels. However, it is hard to achieve synchronization or any type of coordination

\footnotetext{
* Correspondence: jfang1985@gmail.com

NYU WIRELESS, Department of Electrical and Computer Engineering, Polytechnic School of Engineering of New York University, 6 MetroTech
} Center, Brooklyn 11201, USA between PUs and SUs or among SUs since they transmit independently. Therefore, on one hand, waveform shaping for spectral containment by a SU transmitter is needed to minimize its out-of-band emission (OOBE) in order to reduce interference to its neighboring PUs and/ or SUs. On the other hand, an efficient adjacent channel interference $(\mathrm{ACI})$ rejection approach that can reduce the ACI from neighboring PUs and/or SUs is needed at a SU receiver. Although ACI rejection is equally important as OOBE suppression, there are much more work on OOBE suppression than ACI rejection. This may be due to the fact that, for the conventional approaches (windowing and filtering), the principle of ACI rejection is very similar to that of OOBE rejection. However, this is not always true. Take orthogonal frequency division multiplexing (OFDM) as an example. If the ACI rejection

\section{国}


is done before the removal of cyclic prefix $(\mathrm{CP})$ at the OFDM receiver without special considerations, ACI will regenerate because the processed interference signal is also truncated during the $\mathrm{CP}$ removal process. In addition, since OFDM signal is demodulated symbol by symbol, a timing offset (TO) between the desired and interfering transmitters can enhance the equivalent OOBE of the interfering transmitter greatly. These issues have not been addressed so far. Thus, considerations of OOBE suppressions and ACI rejection are not all alike, and it is important to find out which approach is better for OOBE suppression and which approach is better for $\mathrm{ACI}$ rejection.

OFDM technology, which divides the total bandwidth into several orthogonal subbands, provides the flexibility for deactivating specific subcarriers simply by using zeros as the corresponding input values. In addition, OFDM has several favorable properties like high spectrum efficiency, robustness to channel fading, multipath delay spread tolerance, efficient digital signal processing implementation, and granular resource allocation, etc. (for example, in [4, 5]). Because of these merits, OFDM has been considered as a candidate for CR in the first CR-based standard IEEE 802.22 [6]. However, OFDM has shortcomings such as high peak-to-average power ratio (PAPR), high sensitivity to frequency offset (FO) and TO, large inter-carrier interference (ICI) at high Doppler's, and large OOBE and/or ACI originating from large side lobes of the rectangular pulse shape. Among these shortcomings, the high OOBE and/or ACI is the main technical challenge for CR applications (although the other shortcomings may induce OOBE and/or ACI as well).

Various approaches have been proposed to suppress OOBE of OFDM signals. These approaches can be classified into two categories: the time-domain and frequencydomain approaches. The time-domain approaches include windowing [7] and filtering [8] where the OOBE suppression processes are carried out in the time domain (after the inverse fast Fourier transform (IFFT) operation). The frequency-domain approaches include subcarrier weighting [9], carrier cancellation [10, 11], and spectral precoding $[12,13]$, where the OOBE suppression processes are carried out in the frequency domain (before the IFFT operation). As mentioned before, the time-domain OOBE suppression approaches (windowing and filtering) at the transmitter can also be used at the receiver to reject $\mathrm{ACI}$ $[14,15]$. However, it is not obvious that the spectral decoder at the receiver (which corresponds to the frequencydomain spectral precoding at the transmitter for OOBE suppression) can also reject $\mathrm{ACI}$ as well.

Each of the abovementioned approaches has merits and drawbacks. For the time-domain examples, the windowing approach has low complexity and no spectral efficiency loss. It can be used to suppress OOBE or reject $\mathrm{ACI}$ as long as the roll-off portion of the windowing function is long enough. However, a long roll-off portion will induce inter-symbol interferences (ISI) and subsequently seriously degrade the bit error rate (BER) performance if the CP length is not long enough. Therefore, the performance of windowing approach is limited by the $\mathrm{CP}$ length. Like windowing, the filtering approach has no spectral efficiency loss, and its performance is limited by the CP length. However, unlike windowing, it is less sensitive to the TO between PU and SUs when it is used to suppress OOBE of OFDM signal and has high complexity when the spectrum is non-contiguous or dynamically changing. For the frequency-domain example, spectral precoding approach is very efficient for reducing OOBE and can be easily extended to reduce PAPR simultaneously by combining with PAPR precoding [16]. However, it suffers spectral efficiency loss and has high computational complexity when the number of subcarriers is large or when the spectrum is non-contiguous or dynamically changing. In addition, its BER performance will degrade when there is TO between PU and SU. In summary, none of the existing approaches can deal with all practical issues and a study of these approaches under various practical conditions would be very valuable.

However, in most of published works for OOBE/ACI suppression, these existing approaches are evaluated in their favorable conditions (which are not practical) and therefore the suitability of these approaches in practical situations has not been properly addressed. For examples, firstly, in most of publications about the time-domain approaches, the $\mathrm{CP}$ length was oftentimes extended so long that the effects of delay spread is minimized. But the $\mathrm{CP}$ length is fixed in practice (e.g., in Long-Term Evolution (LTE) [17]), and a large delay spread will cause both ISI and ICI if a windowing or filtering approach is employed. Secondly, for the spectral precoding approach, the complexity and implementation issues for broadband channels have not been addressed. But, in reality, the CR system is usually broadband. Thirdly, the effects of other impairments (such as PAPR, TO, FO, etc.) on OOBE or ACI are usually not assessed. However, these impairments may cause significant increases of OOBE and ACI. Fourthly, the implications of non-contiguousness and time variations of the available spectrum on the complexity issues are mostly unaddressed. However, the available spectrum in CR is usually non-contiguous and dynamically changing.

As the frequency-domain approach (precoding) and the two time-domain approaches (windowing and filtering) have complementary characteristics, a hybrid combination of these approaches may be advantageous if it can maintain the merits and mitigate the drawbacks of these approaches. In this paper, hybrid approaches which combine the frequency-domain spectral coding and either one of the two time-domain approaches (windowing and filtering) are proposed to deal with various abovementioned 
practical issues. Detailed analyses are made on the complexity, spectral efficiency, spectral containments, PAPR and BER of the windowing, filtering, and spectral precoding approaches, and their possible combination schemes under practical conditions (broadband channels with radio impairments such as FO and TO). Simulation results show that the proposed hybrid approaches indeed maintain the merits and mitigate the drawbacks of their constituent approaches. Particularly, since windowing process can be carried out conveniently to deal with the CP removal issue, a hybrid combination of precoding and widowing is more appropriate for ACI rejection. On the other hand, since filtering is less sensitive to TO, a hybrid combination of precoding and filtering is more appropriate for OOBE suppression for OFDM systems.

This paper is organized as follows. In Section 2, we present the frequency and time-domain OOBE suppression approaches and the hybrid approaches. In Sections 3 and 4 , we deal with various practical issues and present numerical examples. Conclusion remarks are made in Section 5. The notation of this paper is as follows. All boldface letters indicate vectors (lowercase) or matrices (uppercase). $\boldsymbol{A}^{H}$ and $\boldsymbol{A}^{T}$ stand for the conjugate transpose and transpose of $\boldsymbol{A}$, respectively. $[\boldsymbol{A}]_{i, j}$ denotes the element of $\boldsymbol{A}$ at the $i$ th row and jth column. $a^{*}$ and $E(a)$ denotes the conjugate and expectation of $a$, respectively. diag [...] denotes the diagonal matrix with elements [...] on the main diagonal. $\boldsymbol{I}_{r}$ represents an $r$ by $r$ identity matrix. $a \otimes b$ denotes the convolution of $a$ and $b$.

\section{Formulation}

\subsection{Conventional CP-OFDM transmit signal}

The $l$ th time-domain conventional CP-OFDM transmit symbol $\phi_{l}(t)$ of $N$ contiguous subcarriers can be expressed as:

$$
\phi_{l}(t)=\sum_{n=\mathcal{N}_{0}}^{\mathcal{N}_{0}+N-1} d_{l, n} p_{n}(t-l T), l T \leq t<(l+1) T
$$

where $d_{l, n}$ is the $l$ th data symbol transmitted on the $n$th subcarrier, and

$$
p_{n}(t)=e^{j 2 \pi \frac{n}{T_{\mathrm{s}}} t} g(t)
$$

The pulse shape function

$$
g(t)=\left\{\begin{array}{l}
1,-T_{\mathrm{CP}} \leq t<T_{\mathrm{s}} \\
0, \quad \text { otherwise }
\end{array}\right.
$$

where $T_{\mathrm{s}}$ and $T_{\mathrm{CP}}$ is the symbol and the CP durations, respectively. In (1), $T=T_{\mathrm{CP}}+T_{\mathrm{S}}$ is the effective symbol duration.

\subsection{General framework for OOBE and $\mathrm{ACl}$ suppression approaches}

Both time-domain and frequency-domain approaches can be used to suppress the OOBE at the transmitter and reject the ACI at the receiver. The block diagram in Fig. 1 is used to describe not only the time-domain and frequency-domain approaches but also the hybrid approaches which combine the processes in both frequency and time domains.

\subsubsection{OOBE suppression at the transmitter}

For the frequency-domain OOBE suppression approach at the transmitter, the precoded data vector $\boldsymbol{s}_{l}=\left[s_{l, \mathcal{N}_{0}}, \ldots, s_{l, \mathcal{N}_{0}+N-1}\right]^{T}$ with $N$ correlated elements is obtained by precoding the $M(M \leq N)$ uncorrelated elements of the original data vector $\boldsymbol{d}_{l}=\left[d_{l, \mathcal{N}_{0}}, \ldots, d_{l, \mathcal{N}_{0}+M-1}\right]^{T}$ by a semi-unitary precoding matrix $\boldsymbol{G}$ with dimension of $(N \times M)$,

$$
\boldsymbol{s}_{l}=\boldsymbol{G d}_{l}, \quad \text { with } \boldsymbol{G}^{\boldsymbol{H}} \boldsymbol{G}=\boldsymbol{I}_{M}
$$

Instead of $\boldsymbol{d}_{l}, \boldsymbol{s}_{l}$ is fed into IFFT to generate the $l$ th OFDM symbol. Note that, in essence, only $M$ data elements are transmitted per OFDM symbol in the available frequency band, reserving $N-M$ degrees of freedom for shaping the spectrum of the transmitted signal.

For the time-domain OOBE suppression approach at the transmitter, either a bandpass filter $f_{\mathrm{tx}}(t)$ or a windowing function $w_{\mathrm{tx}}(t)$ is employed and the $l$ th transmit signal is

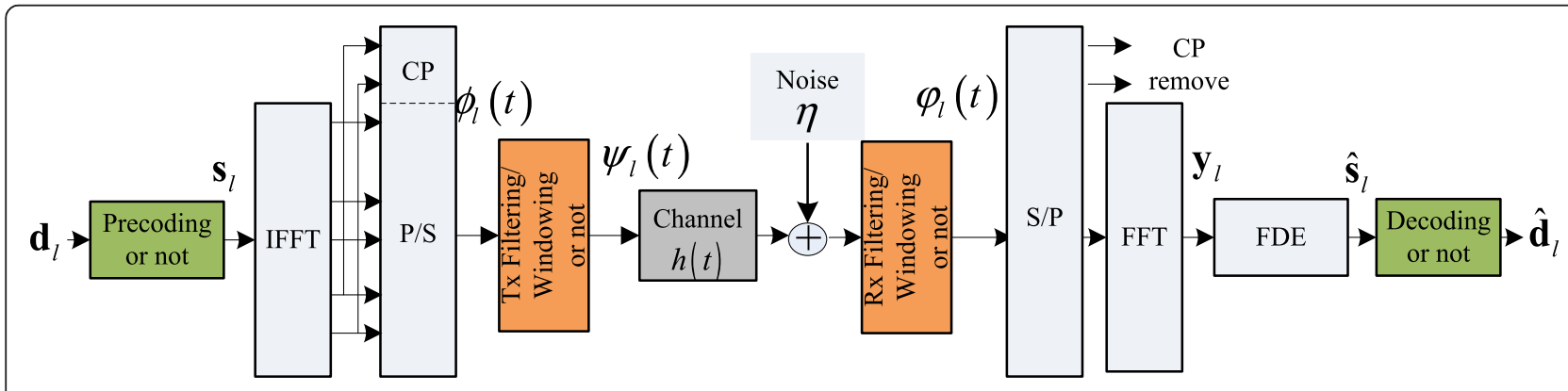

Fig. 1 A block diagram of the multicarrier modulation transceiver 


$$
\psi_{l}(t)=\left\{\begin{array}{cl}
\phi_{l}(t) \otimes f_{\mathrm{tx}}(t) & \text { filtering } \\
\phi_{l}(t) w_{\mathrm{tx}}(t-l T) & \text { windowing }
\end{array}\right.
$$

\subsubsection{Channel and equalizer}

The $l$ th signal received at the receiver is

$$
z_{l}(t)=\psi_{l}(t) \otimes h(t)+\eta(t),
$$

where $h(t)$ is the channel impulse response and $\eta(t)$ is the additive white Gaussian noise with power spectral density (PSD) $N_{0}$. Considering a filtering or windowing process at the front end of the receiver as shown in Fig. 1, the resultant signal is

$$
\phi_{l}(t)=\phi_{l}(t) \otimes h_{\mathrm{eff}}(t)+\eta_{\mathrm{eff}}(t)
$$

where $h_{\text {eff }}$ and $\eta_{\text {eff }}$ are effective channel (see (12)) and effective noise (see (13)), respectively. If the CP duration $T_{\mathrm{CP}}$ is larger than the delay spread of the effective channel, the symbol can be estimated at the receiver without ISI. Using (4), the data $y_{l}$ can be written as

$$
\boldsymbol{y}_{l}=\boldsymbol{H} \boldsymbol{G} d_{l}+\Theta=H s_{l}+\Theta
$$

where

$$
\begin{aligned}
& \boldsymbol{y}_{l}=\left[y_{l, 0}, y_{l, 1}, \ldots, y_{l, N-1}\right]^{T} \\
& \boldsymbol{H}=\operatorname{diag}\left[H_{0}, H_{1}, \ldots, H_{N-1}\right], H_{n}=\int_{0}^{T_{\mathrm{s}}} h_{\mathrm{eff}}(t) e^{-j 2 \pi} \frac{n}{T_{\mathrm{s}}} t d t \\
& \boldsymbol{\Theta}=\left[\Theta_{0}, \Theta_{1}, \ldots, \Theta_{N-1}\right]^{T}, \Theta_{n}=\int_{0}^{T_{\mathrm{s}}} \eta_{\mathrm{eff}}(t) e^{-j 2 \pi} \frac{n}{T_{\mathrm{s}}} t d t
\end{aligned}
$$

If a minimum mean square error (MMSE) frequencydomain equalizer (FDE) is used, the estimated $\hat{s}_{l}$ can be obtained by

$$
\hat{s}_{l}=\boldsymbol{D}_{\mathrm{MMSE}} \boldsymbol{y}_{l},
$$

where

$$
\boldsymbol{D}_{\mathrm{MMSE}}=E\left\{\boldsymbol{s}_{\boldsymbol{l}} \boldsymbol{s}_{l}{ }^{H}\right\}(\hat{\boldsymbol{H}})^{H}\left(\hat{\boldsymbol{H}} E\left\{\boldsymbol{s}_{l} \boldsymbol{s}_{l}{ }^{H}\right\}(\hat{\boldsymbol{H}})^{\boldsymbol{H}}+E\left\{\boldsymbol{\Theta} \Theta^{H}\right\}\right)^{-1}
$$

\subsubsection{ACl rejection at the receiver}

For the time domain ACI rejection approach at the receiver, either a bandpass filter $f_{\text {rx }}(t)$ or a windowing function $w_{\mathrm{rx}}(t)$ is employed, the effective channel in (7) is

$$
h_{\mathrm{eff}}(t)=\left\{\begin{array}{cc}
h(t) \otimes f_{\mathrm{tx}}(t) \otimes f_{\mathrm{rx}}(t) & \text { filtering } \\
h(t) w_{\mathrm{tx}}(t-l T) w_{\mathrm{rx}}(t-l T) & \text { windowing }
\end{array}\right.
$$

and the effective noise in (7) is

$$
\eta_{\mathrm{eff}}(t)=\left\{\begin{array}{cl}
\eta(t) \otimes f_{\mathrm{rx}}(t) & \text { filtering } \\
\eta(t) w_{\mathrm{rx}}(t-l T) & \text { windowing }
\end{array}\right.
$$

When filters and windowing functions are used at the receiver as the time-domain process to reject $\mathrm{ACI}$, the effective noise PSD in (11) are

$$
\begin{aligned}
E\left(\Theta_{n} \Theta_{n}^{*}\right) & =\left|F_{r x}(n)\right|^{2} N_{0}, F_{r x}(n)=\int_{0}^{T_{s}} f_{r x}(t) e^{-j 2 \pi} \frac{n}{T_{\mathrm{s}}} t d t \text { and } \\
E\left(\Theta_{n} \Theta_{n}^{*}\right) & =N_{0} \int_{0}^{T_{s}}\left|w_{r x}(t)\right|^{2} d t,
\end{aligned}
$$

respectively.

The frequency-domain ACI rejection at the receiver, corresponding to the OOBE suppression at the transmitter, is implemented using $\boldsymbol{G}^{H}$ and the estimated $\hat{\boldsymbol{d}}_{l}$ can be obtained by the hard decision made after decoding

$$
\hat{\boldsymbol{d}}_{l}=\operatorname{decision}\left\{\boldsymbol{G}^{H} \hat{\boldsymbol{s}}_{l}\right\}
$$

Let $E\left(\boldsymbol{d}_{l} \boldsymbol{d}_{l}^{H}\right)=E_{0} \boldsymbol{I}_{M}$ where $E_{0}=E\left(d_{l, m} d_{l, m}^{*}\right)$, for every $l$ and $m$, is the data energy. With (4), $\boldsymbol{D}_{\mathrm{MMSE}}$ in (11) can be written as:

$$
\boldsymbol{D}_{\mathrm{MMSE}}=E_{0}\left(\boldsymbol{G} \boldsymbol{G}^{\boldsymbol{H}}\right)(\hat{\boldsymbol{H}})^{\boldsymbol{H}}\left(\hat{\boldsymbol{H}}\left(\boldsymbol{G} \boldsymbol{G}^{\boldsymbol{H}}\right)(\hat{\boldsymbol{H}})^{\boldsymbol{H}} E_{0}+E\left\{\boldsymbol{\Theta} \boldsymbol{\Theta}^{\boldsymbol{H}}\right\}\right)^{-1}
$$

\subsubsection{Various approaches}

The block diagram in Fig. 1 is a general framework for not only the hybrid approaches but also the individual time or frequency-domain approaches. When filters are employed at both the transmitter and receiver as the time-domain process, the hybrid approach is named filtered-precoded-OFDM (F-P-OFDM). Similarly, when windowing functions are employed at both the transmitter and receiver as the time-domain process, the hybrid approach is named windowed-precoded-OFDM (W-POFDM). If there is no time-domain process,

$$
h_{\text {eff }}(t)=h(t) \text { and } \eta_{\text {eff }}(t)=\eta(t)
$$

the hybrid approaches (F-P-OFDM and W-P-OFDM) are reduced to precoded-OFDM (P-OFDM). If there is no frequency-domain process,

$$
\boldsymbol{G}=\boldsymbol{I}_{N}
$$

the hybrid approach F-P-OFDM is reduced to filteredOFDM (F-OFDM) and the hybrid approach W-POFDM is reduced to windowed-OFDM (W-OFDM). If both (17) and (18) are satisfied and neither time or frequency-domain process is employed, the approaches are reduced to the conventional CP-OFDM. Due to the constraint on paper length, many other possible combinations are not included in this paper. 


\subsection{Frequency-domain approaches: P-OFDM}

Two different precoding techniques, spectral precoding and PAPR precoding, are employed to suppress OOBE and PAPR, respectively. In order to distinguish these two precoded-OFDM (P-OFDM), they are named as spectral precoded (SP)-OFDM and PAPR precoded (PP)-OFDM in this paper. Our research in [16] shows that these two precoding techniques can be combined to achieve satisfactory spectral containments and PAPR performance simultaneously. (The spectrum containment of a waveform is satisfactory when the stopband attenuation is larger than $55 \mathrm{~dB}$, as in the spectral mask requirement of Federal Communications Commission (FCC) TV white space when the effective isotropic radiated power is $+36 \mathrm{dBm}$ [18].) The combination of SP-OFDM and PP-OFDM will be denoted as PMix-OFDM. Also, for convenience, let POFDM represent general precoded-OFDM which includes PP-OFDM, SP-OFDM, and PMix-OFDM.

The main idea of spectral precoding $G \mathbf{s}$ is to project the $M$-dimensional data symbol vector, $\boldsymbol{d}_{l}$, into an $N$-dimensional linear subspace [19] that ensures the desired spectral containment. Denote the frequency-domain representation of $\phi_{l}(t)$ in (1) at frequency $f$ as

$$
X(f)=\sum_{n=0}^{N-1} s_{l, n} P_{n}(f)
$$

with

$$
\begin{aligned}
P_{n}(f) & =\frac{1}{T} \int_{-T_{C P}}^{T_{s}} p_{n}(t) e^{-j 2 \pi f t} d t \\
& =\frac{e^{j \pi\left(\frac{n}{T_{s}}-f\right)\left(T_{s}-T_{C P}\right)}}{\pi\left(\frac{n}{T_{s}}-f\right) T} \sin \left(\pi\left(\frac{n}{T_{s}}-f\right) T\right)
\end{aligned}
$$

Using matrix expressions, the frequency responses $\boldsymbol{x}$ at $K$ selected out-of-band frequencies (called notched frequencies), $\left\{f_{k}, k=0,1, \ldots, K-1\right\}$, can be written as

$$
\begin{aligned}
\boldsymbol{x}=\boldsymbol{P} \boldsymbol{s}_{l}=\boldsymbol{P} G_{s} \boldsymbol{d}_{l}, \boldsymbol{x} & =\left[\begin{array}{c}
X\left(f_{0}\right) \\
X\left(f_{1}\right) \\
\vdots \\
X\left(f_{K-1}\right)
\end{array}\right], \\
\boldsymbol{P} & =\left[\begin{array}{ccc}
P_{0}\left(f_{0}\right) & \cdots & P_{N-1}\left(f_{0}\right) \\
\vdots & \ddots & \vdots \\
P_{0}\left(f_{K-1}\right) & \cdots & P_{N-1}\left(f_{K-1}\right)
\end{array}\right]
\end{aligned}
$$

OOBE suppression can then be done by projecting $\boldsymbol{d}_{l}$ into a linear subspace with the form:

$$
\mathcal{L}(\boldsymbol{P})=\left\{\boldsymbol{s}_{l} \mid \boldsymbol{P} \boldsymbol{s}_{l}=\mathbf{0}\right\}
$$

The solution to this problem is to use a semi-unitary precoding matrix $G \mathbf{s}$ whose columns constitute an orthogonal basis of $\mathcal{L}(\boldsymbol{P})$. Performing the singular value decomposition (SVD) on $\boldsymbol{P}$ :

$$
\boldsymbol{P}=\boldsymbol{U} \boldsymbol{\Sigma} \boldsymbol{V}^{H}
$$

where $\boldsymbol{U}$ is a $K \times K$ unitary matrix, $\boldsymbol{\Sigma}$ is a $K \times N$ diagonal matrix containing the singular values of $\boldsymbol{P}$ in non-increasing order on its diagonal and $V$ is an $N \times N$ unitary matrix whose columns are $\boldsymbol{v}_{0}, \boldsymbol{v}_{1}, \cdots, \boldsymbol{v}_{N-1}$. The last $M$ columns of $V$ are collected to construct the $N \times M$ precoding matrix

$$
\boldsymbol{G}_{\mathrm{S}}=\left[\begin{array}{lll}
\mathbf{v}_{N-M+1} \cdots & \mathbf{v}_{N-2} & \mathbf{v}_{N-1}
\end{array}\right]
$$

Define $\lambda=\frac{M}{N}$ as the coding rate and $R=N-M$ as the coding redundancy. Better spectral containments can be achieved at the cost of higher coding redundancy. If $R \geq K$, $\boldsymbol{x}=0$ for any arbitrary data vector $\boldsymbol{d}_{l}$ due to the fact that $\boldsymbol{s}_{l}$ is in the null space of $\boldsymbol{P}$. In this paper, we choose $R \leq K$.

The main idea of PAPR precoding is to project the $M$ dimensional data symbol vector $\boldsymbol{d}_{l}$ into a $N$-dimensional linear subspace that ensures the desired PAPR performance. The transmit signal in (1) can be rewritten as:

$$
\phi_{l}(t)=\sum_{m=\mathcal{N}_{0}}^{\mathcal{N}_{0}+M-1} d_{l, m} p_{m}^{\prime}(t-l T)
$$

where $p_{m}^{\prime}(t)$ is the inverse discrete Fourier transform (IDFT) of the $m$ th column of the precoder $\boldsymbol{G}_{\mathrm{PAPR}}$

$$
p_{m}^{\prime}(t) \equiv \sum_{n=0}^{N-1} G_{n, m} e^{j \frac{2 \pi n}{T_{s}} t} .
$$

Note that the $m$ th data $d_{l, m}$ is carried by the waveform $p_{m}^{\prime}(t)$. Then, to suppress the PAPR, we can choose $\left\{G_{n, m}\right\}$ such that the peaks of different $p_{m}^{\prime}(t)$ for different $m$ occurs at different times within an OFDM symbol. The waveform design has been investigated in [20,21], and goes beyond the scope of this paper. For simplicity, we use the discrete Fourier transform (DFT) precoding matrix $\boldsymbol{G}_{\mathrm{DFT}}$ in [22] with elements:

$$
\left[\boldsymbol{G}_{\mathrm{PAPR}}\right]_{k, n}=\left[\boldsymbol{G}_{\mathrm{DFT}}\right]_{k, n}=\frac{1}{\sqrt{N}} e^{-j \frac{2 \pi k}{N} n}
$$

which makes different $p_{m}^{\prime}(t)$ as sinc functions with their peaks at different times.

Note that, the precoding matrix in (24) can be easily extended to reduce the PAPR by multiplying the spectral precoding matrix $\boldsymbol{G}_{\mathrm{S}}$ by the DFT matrix $\boldsymbol{G}_{\mathrm{DFT}}$ :

$$
\boldsymbol{G}_{\mathrm{Mix}}=\boldsymbol{G}_{\mathrm{S}} \boldsymbol{G}_{\mathrm{DFT}}
$$

\subsection{Time-domain approaches: F-OFDM and W-OFDM}

Two main time-domain OOBE suppression approaches are summarized here. For F-OFDM, to suppress the OOBE at the transmitter, the CP-OFDM signal is convolved with a transmit filter, $f_{\mathrm{tx}}(t)$, with a length of $\alpha_{\mathrm{tx}} T_{\mathrm{s}}$, where $\alpha_{\mathrm{tx}}$ is the normalized transmit filter length, and to 
reject the ACI at the receiver, the received signal is convolved with a receive filter, $f_{\mathrm{tx}}(t)$, with a length of $\alpha_{\mathrm{rx}} T_{\mathrm{s}}$, where $\alpha_{\mathrm{rx}}$ is the normalized receive filter length. For convenience, an identical filter is used at both the transmitter and receiver, i.e., $f_{\mathrm{tx}}(t)=f_{\mathrm{rx}}(t)$. An example of the identical filter is a square root raised cosine filter. For W-OFDM, to suppress OOBE at the transmitter, the CP-OFDM signal is point-to-point multiplied with a transmit windowing function, $w_{\mathrm{tx}}(t)$, before transmission, and to reject $\mathrm{ACI}$ at the receiver, the received signal is point-to-point multiplied with a receive windowing function, $w_{\mathrm{rx}}(t)$. An example of $w_{\mathrm{tx}}(t)$ is given below:

$$
w_{\mathrm{tx}}(t)=\left\{\begin{array}{lr}
0.5\left(1+\cos \left\{\pi\left(1+\frac{t}{\beta_{\mathrm{tx}} T_{\mathrm{s}}}\right)\right\}\right), & 0 \leq t<\beta_{\mathrm{tx}} T_{\mathrm{s}} \\
1, & \beta_{\mathrm{tx}} T_{\mathrm{s}} \leq t<T_{\mathrm{s}}+T_{\mathrm{CP}} \\
0.5\left(1+\cos \left\{\pi\left(\frac{t-T_{\mathrm{s}}}{\beta_{\mathrm{tx}} T_{\mathrm{s}}}\right)\right\}\right), T_{\mathrm{s}}+T_{\mathrm{CP}} \leq t \leq\left(1+\beta_{\mathrm{tx}}\right) T_{\mathrm{s}}+T_{\mathrm{CP}}
\end{array}\right.
$$

where $\beta_{\mathrm{tx}} T_{\mathrm{s}}$ is the roll-off portion of $w_{\mathrm{tx}}(t)$. As shown in Fig. 2, the CP-OFDM signal is extended with CP and cyclic suffix (CS), then is point-to-point multiplied with the windowing function $w_{\mathrm{tx}}(t)$. In order to keep the symbol duration of OFDM symbol unchanged after windowing, the ending $\beta_{\mathrm{tx}} T_{\mathrm{s}}$ portion of the current windowed-OFDM symbol overlaps with the beginning $\beta_{\mathrm{tx}} T_{\mathrm{s}}$ portion of the next windowedOFDM symbol.

At the receiver, as the CP is discarded before FFT to reduce ISI, the windowing process should be carried out on the signal portion that goes through the FFT for rejecting ACI. Thus, an example of the windowing function is:

$$
w_{\mathrm{rx}}(t)=\left\{\begin{array}{cr}
0, & 0 \leq t<T_{\mathrm{CP}}-\beta_{\mathrm{rx}} T_{\mathrm{s}} \\
0.5\left(1+\cos \left\{\pi\left(1+\frac{t}{\beta_{\mathrm{rx}} T_{\mathrm{s}}}\right)\right\}\right), & T_{\mathrm{CP}}-\beta_{\mathrm{rx}} T_{\mathrm{s}} \leq t<T_{\mathrm{CP}} \\
1, & T_{\mathrm{CP} \leq t \leq\left(1-\beta_{\mathrm{rx}}\right) T_{\mathrm{s}}+T_{\mathrm{CP}}} \\
0.5\left(1+\cos \left\{\pi\left(\frac{t-T_{\mathrm{s}}}{\beta_{\mathrm{rx}} T_{\mathrm{s}}}\right)\right\}\right), & \left(1-\beta_{\mathrm{rx}}\right) T_{\mathrm{s}}+T_{\mathrm{CP}}<t \leq T_{\mathrm{s}}+T_{\mathrm{CP}}
\end{array}\right.
$$

where $\beta_{\mathrm{rx}} T_{\mathrm{s}}$ is the roll-off duration (see Fig. 3). Note that, in order to keep the orthogonality of OFDM symbol, the windowing function is chosen to satisfy

$$
w_{\mathrm{rx}}(t)+w_{\mathrm{rx}}\left(t+T_{\mathrm{s}}\right)=1, T_{\mathrm{CP}}-\beta_{\mathrm{rx}} T_{\mathrm{s}} \leq t<T_{\mathrm{CP}}
$$

so that, as shown in Fig. 3, the roll-off section of OFDM symbol after windowing can be added back to the tail section (the last $\beta_{\mathrm{rx}} T_{\mathrm{s}}$ ) of the OFDM symbol before FFT.

\subsection{Hybrid approaches: F-P-OFDM and W-P-OFDM}

According to Fig. 1, the F-P-OFDM can be simply constructed by using the filters presented in Section 2.4 and the spectral decoder presented in Section 2.3. Similarly, the W-P-OFDM can be simply constructed by using the window functions presented in Section 2.4 and the spectral decoder presented in Section 2.3.

\section{Practical issues and numerical examples: without $\mathrm{ACl}$}

Various practical issues including non-contiguous and dynamically changing spectrum, multipath delay spread, radio impairments (FO and $\mathrm{TO}$ ), implementation complexity, spectral efficiency, PAPR, spectral containment, interference rejection, etc., will be addressed in this section and Section 4. The performances of CP-OFDM (without any OOBE or ACI suppression approach), POFDM, F-OFDM, W-OFDM, F-P-OFDM, and W-P-

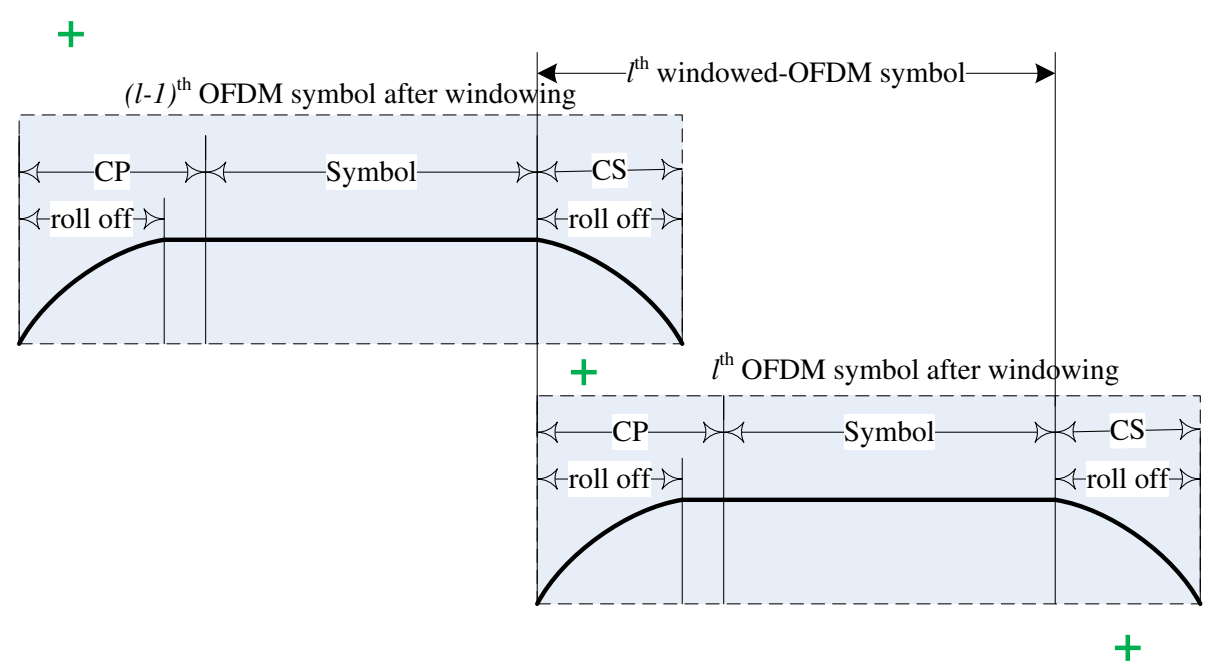

Fig. 2 Illustration of a windowed-OFDM transmitted symbol 


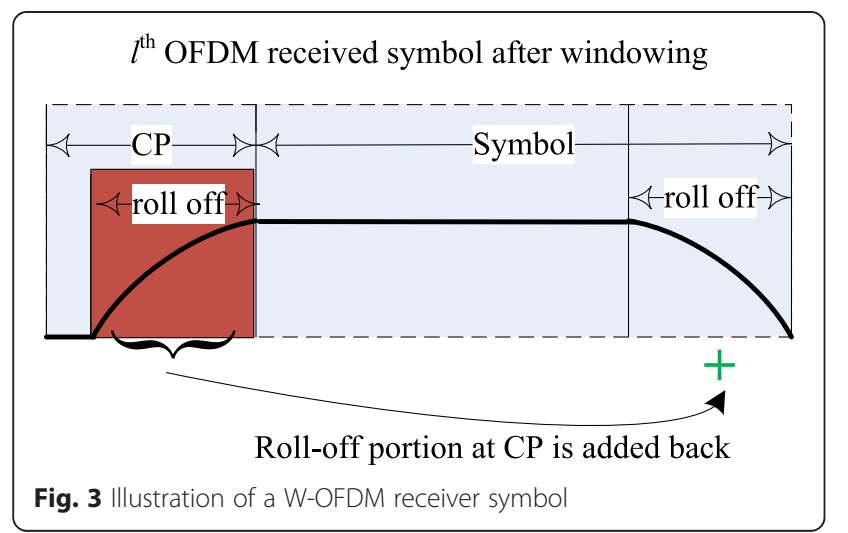

OFDM are evaluated in terms of complexity, coding rate, spectral containment, PAPR, and BER based on a LTE-like system. Specifically, results shown in this section are without ACI and results shown in Section 4 are with ACI. The simulation parameters are specified in Table 1, if not otherwise indicated.

\subsection{Complexity and coding rate analysis}

In this subsection, the coding rates and computational complexities of various OOBE suppression or ACI rejection approaches are presented. The analysis of coding rate $\lambda$ is straight forward. For approaches involving precoding (P-OFDM, F-P-OFDM, and W-P-OFDM), their coding rates are $1-R / N$. However, for approaches without precoding (CP-OFDM, F-OFDM, and W-OFDM), their coding rates are 1 . The analysis of complexity is much more involved. Here, the computational complexity for each approach is evaluated in terms of the number of real multiplications needed for completing the transmission of one quadrature amplitude modulation (QAM) input symbol vector $\boldsymbol{d}_{l}$ from the transmitter to the receiver. But the multiplications with \pm 1 and $\pm j$ are not included since they are merely flips of sign and/or flips of real and imaginary parts. In addition, since the channel and equalizer coefficients are assumed known,

Table 1 LTE-like systems

\author{
System parameters \\ Symbol duration: $T_{s}=1 / \Delta f$ \\ Subcarrier spacing: $\Delta f=15 \mathrm{kHz}$ \\ Number of samples per symbol duration: $L=1024$ \\ Active subcarrier index $=[-300:-1,1: 300]$ \\ CP parameters \\ One subframe: 2 slots/subframe
}

One slot: 7 data symbols ( $7 T_{\mathrm{s}}$ and half additional symbol duration (0.5Ts is used for $\mathrm{CP}$, with $\mathrm{CP}$ duration $T_{\mathrm{cp} 1}=80 T_{\mathrm{s}} / 1024 \mathrm{~L}_{\mathrm{cp} 1}=80 \mathrm{~L} / 1024$ for the first symbol and $T_{\mathrm{cp} 2}=72 T_{\mathrm{s}} / 1024 \mathrm{~L}_{\mathrm{cp} 2}=72 \mathrm{~L} / 1024$ for the other symbols.

Average CP length: $L_{c p}=L / 14$ the computational efforts for channel estimation and equalizer design are not included.

Note that the complexity of an $L$-point FFT/IFFT (via split radix FFT [23]) with $L$ complex inputs is $L \log _{2} L-$ $3 L+4$. And the complexity of a scalar 1-tap MMSE FDE equalization is $4 N$ for $N$ subcarriers. Therefore, the complexity of the CP-OFDM without any OOBE or ACI suppression approach is $2\left(L \log _{2} L-3 L+4\right)+4 N$.

For P-OFDM, including SP-OFDM, PP-OFDM, and PMix-OFDM, the complexity of precoding and decoding is $8 N(N-R)$. For a matrix MMSE FDE, the complexity is $4 N^{2}$ since a matrix product is required. Thus, including the complexity for IFFT and FFT, the complexity for POFDM is $2\left(L \log _{2} L-3 L+4\right)+8 N(N-R)+4 N^{2}$, which is proportional to $N^{2}$ if $N$ is large.

For F-OFDM, it is assumed that the transmit filtering and addition of the $\mathrm{CP}$ could be combined such that the filtering is only done once for the CP samples [24]. Therefore, the complexity of transmit filtering is $2 L L \alpha_{\mathrm{tx}}$. But the filtering at the receiver is done for all symbol duration. Therefore, the complexity of receive filtering is $2\left(L+L_{\mathrm{CP}}\right) L \alpha_{\mathrm{rx}}$. Thus, including the complexity of IFFT and FFT, the complexity of F-OFDM with a scalar 1-tap MMSE FDE is $2\left(L \log _{2} L-3 L+4\right)+2 L^{2} \alpha_{\mathrm{tx}}+2(L+$ $\left.L_{\mathrm{CP}}\right) L \alpha_{\mathrm{rx}}+4 N$. Note that, the complexity increases as $\alpha_{\mathrm{tx}}$ or $\alpha_{\mathrm{rx}}$ increases.

For W-OFDM, the complexity of point-to-point multiplication of CP-OFDM signal and windowing at the transmitter and receiver is $2 L \beta_{\mathrm{tx}}$ and $2 L \beta_{\mathrm{rx}}$, respectively, which are relatively small. Including the complexity of IFFT and FFT, the complexity of for W-OFDM with a scalar 1-tap MMSE FDE is $2\left(L \log _{2} L-3 L+4\right)+2 L\left(\beta_{\mathrm{tx}}+\beta_{\mathrm{rx}}\right)+4 N$. Therefore, the complexity of W-OFDM is very close to that of CP-OFDM.

For F-P-OFDM, including F-SP-OFDM, F-PP-OFDM, and F-PMix-OFDM, the complexity, including IFFT/FFT, filtering, precoding, decoding and equalization using a matrix MMSE FDE, is $2\left(L \log _{2} L-3 L+4\right)+2 L L \alpha_{t x}+$ $2\left(L+L_{\mathrm{CP}}\right) L \alpha_{\mathrm{rx}}+8 N(N-R)+4 N^{2}$ which is proportional to $N^{2}$ if $N$ is large.

For W-P-OFDM, including W-SP-OFDM, W-PP-OFDM and W-PMix-OFDM, the complexity, including IFFT/FFT, windowing, precoding, decoding and equalization using a matrix MMSE FDE, is $2\left(L \log _{2} L-3 L+4\right)+2 L\left(\beta_{\mathrm{tx}}+\beta_{\mathrm{rx}}\right)+$ $8 N(N-R)+4 N^{2}$ which is proportional to $N^{2}$ if $N$ is large.

Table 2 shows the per-symbol normalized complexities (with respect to the complexity of CP-OFDM) of various approaches for $N=60$ and 300. As discussed before, for W-OFDM, the complexity is very close to that of CPOFDM no matter how many subcarriers are used. For FOFDM with the fixed subcarrier number, the complexity increases as the filter length increases and are almost independent of the number of subcarriers. Therefore, for low complexity, a filter with a minimum length is 
Table 2 Normalized complexity (with respect to CP-OFDM) for $N=60$ and 300. Note that P-OFDM represents SP-OFDM, PP-OFDM, or PMix-OFDM and W/F-P-OFDM represents W/F-SP-OFDM, W/F-PP-OFDM, or W/F-PMix-OFDM

\begin{tabular}{llll}
\hline$L=1024$ & & $N=60$ & $N=300$ \\
\hline CP-OFDM & & 1 & 1 \\
P-OFDM & $R=3$ or $R=6$ & $3.85(R=3)$ & $69.48(R=6)$ \\
& $R=6$ or $R=12$ & $3.75(R=6)$ & $68.55(R=12)^{1}$ \\
& & & $34.74(R=12)^{2}$ \\
& & & $23.47(R=12)^{3}$ \\
W-OFDM & $\beta_{\mathrm{tx}}=\beta_{\mathrm{rx}}=24 / L$ & 1.01 & 1.01 \\
& $\beta_{\mathrm{tx}}=\beta_{\mathrm{rx}}=48 / L$ & 1.01 & 1.01 \\
F-OFDM & $a_{\mathrm{tx}}=a_{\mathrm{rx}}=18 / L$ & 6.24 & 5.91. \\
& $a_{\mathrm{tx}}=a_{\mathrm{rx}}=35 / L$ & 11.18 & 10.55 \\
F-P-OFDM & $R=3$ or $6, a_{\mathrm{tx}}=a_{\mathrm{rx}}=18 / L$ & $9.08(R=3)$ & $74.39(R=6)$ \\
W-P-OFDM & $R=3$ or $6, \beta_{\mathrm{tx}}=\beta_{\mathrm{rx}}=24 / L$ & $3.85(R=3)$ & $69.48(R=6)$ \\
\hline
\end{tabular}

desired if OOBE and ACI can be kept within the specs. For precoding related approaches, the complexities of $\mathrm{P}$ OFDM, F-P-OFDM, and W-P-OFDM for $N=300$ are very high because they are proportional to $N^{2}$ when $N$ is large. Fortunately, the complexity can be reduced by dividing the big spectrum block into several smaller spectrum blocks. For example, for $N=300$, the normalized complexity of P-OFDM is 68.55 for one spectrum block (which requires one precoder/decoder pair for 300 subcarriers). The normalized complexity is reduced to 34.74 (or 23.47) for two (or three) spectrum blocks where we use two (or three) precoder/decoder pairs, and each pair is designed to precode/decode 150 (or 100) subcarriers. (The three cases of one, two, and three spectrum blocks are denoted by the superscripts 1,2 , and 3 in Table 2, respectively.) However, the reduction of complexity may cause degradation in spectral containment if the coding rate is kept unchanged (an example will be discussed in the next subsection).

\subsection{Implications for non-contiguous and dynamic changing spectrum}

In $\mathrm{CR}$, the available bandwidth may be non-contiguous and dynamically changing. Among the approaches discussed in Section 2, only the windowing approach can adapt to the changes or the non-contiguousness of the frequency bands easily. However, the performance of windowing is limited by the constraint on the CP length and is not always satisfactory as shown in the simulation result in Section 4. For the filtering and precoding approaches, the computational complexity is high if a single pair of transmit/receive filters or precoder/decoder is employed to deal with the entire possible band (which includes all possible stopbands and passbands). In addition, the design of filters or precoder/decoder has to change whenever the available spectrum changes. To deal with these problems caused by non-contiguous and dynamically changing spectrum, a resource block (RB) strategy for the filtering approach is proposed in [14]. Similarly, we have also proposed a RB strategy for the precoding approach (see Appendix and [25]) to adapt to the non-contiguous and dynamically changing spectrums.

\subsection{Spectral containment and PAPR}

In this subsection, PSD and PAPR of various approaches based on the parameters defined in Tables 1 and 2 with $N=300$ are evaluated and compared. In the legends of Figs. 4 and 5: for $\mathrm{F}(k), k$ is the transmitter filter length in samples; for $\mathrm{W}(k), k$ is the roll-off length of transmit windowing function in samples; for $\operatorname{SP}(R)$, only spectral precoding is used and $R$ is the redundancy length of spectral precoding; and for PMix $(R)$, the combination of spectral precoding and PAPR precoding in (28) is used and $R$ is the redundancy length of the spectral precoding.

For F-OFDM, as shown in Fig. 4, the longer the filter is, the larger the stopband attenuation can be achieved. For W-OFDM, similar to F-OFDM, better spectral containments can be achieved at the cost of a longer roll-off portion of the windowing function. However, W-OFDM has a slower fall-off rate (i.e., larger transition bands) than CP-OFDM (denoted as "None"; representing the case where there is no OOBE suppression process at the transmitter), as shown in Fig. 4. We aim to use a filter with the longest filter length or a windowing function with the longest possible roll-off portion length to reduce inter-user interference as long as the performance loss caused by ISI is below a threshold. For SP-OFDM, the higher the spectral efficiency loss (i.e., the smaller coding rate) is, the larger the stopband attenuation can be achieved. Therefore, better spectral containment is at the cost of higher spectral efficiency loss. For the given examples, comparing with SP, filtering has a slower falloff rate near the edges of the passband but a smaller PSD in the stopband away from the edges, and windowing has a slower fall-off rate and a larger fall-off PSD in the stopband away from the edges.

Generally, a hybrid approach outperforms its individual constituent approaches. For example, F(18)-SP(6)OFDM is better than SP(6)-OFDM and F(18)-OFDM in terms of spectral containment. Furthermore, it is less sensitive to ISI compared with F(35)-OFDM. Similar observations can also be made for W(24)-SP(6)-OFDM. Furthermore, comparing PMix with SP, it is remarkable that the spectral containment performances of PMixOFDM, F-PMix-OFDM, and W-PMix-OFDM are almost the same as (or even better than) those of SP-OFDM, FSP-OFDM, and W-SP-OFDM, respectively. Thus, we 


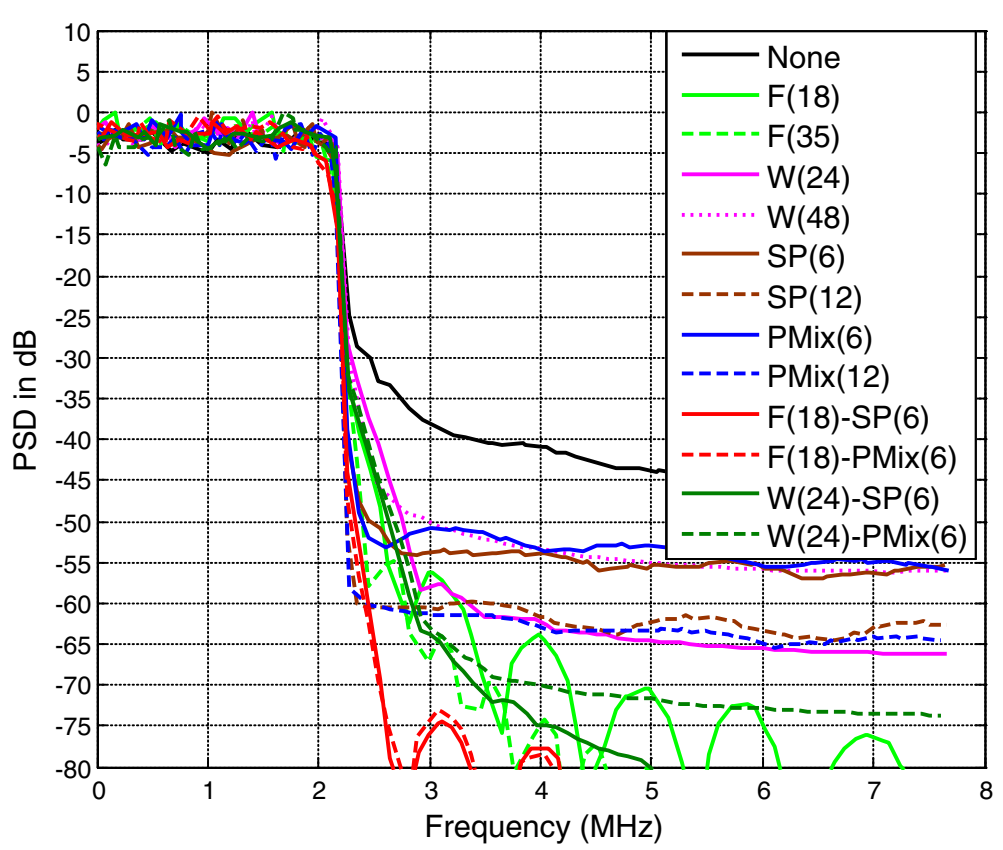

Fig. 4 Spectral containments. In the legends, for $F(k), k$ is the filter length; for $W(k), k$ is the windowing roll-off length; and for $S P(R)$ and $P M i x(R)$, $R$ is the redundancy length of the spectral precoding

conclude that PMix outperforms SP in spectral containment and PAPR (to be shown in Fig. 5).

The effects of RB on PSD will be briefly discussed below. Note that the PSD curve for SP(12)-OFDM in Fig. 4 is derived by using one precoder to precode the entire spectrum block of 300 subcarriers where the 12 redundancies are employed to nullify the frequency responses at 12 notched frequencies (in which, six in the upper stopband and six in the lower stopband). For the two-precoder case discussed in the previous subsection, the passband is divided into two blocks and each precoder is to precode a spectrum block of 150 subcarriers. In this case, the precoder for the upper spectrum block has six notched frequencies in the upper stopband and zero notched frequencies in the lower stopband and the precoder for the lower frequency block has six notched frequencies in the lower stopband and zero notched frequencies in the upper stopband. The PSD result of

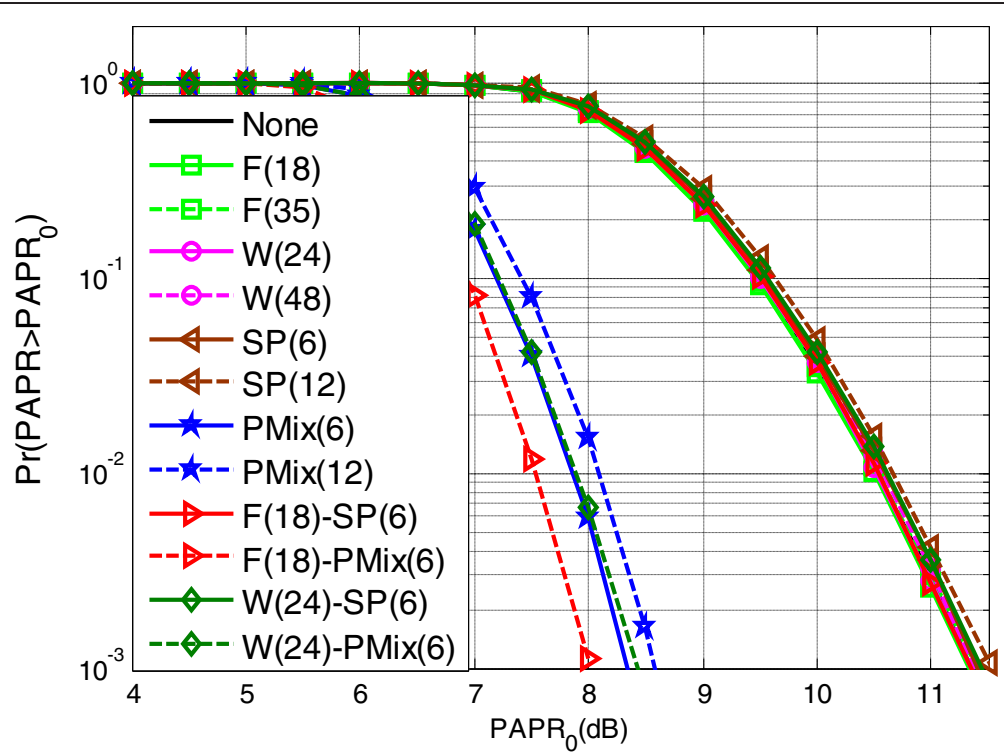

Fig. 5 PAPR performances. The meanings of the legends are the same as those in Fig. 4 
this case is essentially the same as that shown in Fig. 4 (i.e., the one precoder for the entire spectrum block of 300 subcarriers). There is no degradation in spectrum containment, but the normalized complexity is reduced from 68.55 to 34.74 as shown in Table 2. For the three-precoder case which is also discussed in the previous subsection, the OOBE increases $5 \mathrm{~dB}$ if the redundancy number $R$ is kept at 12 . Thus, the reduction of the normalized complexity from 68.55 to 23.47 , as shown in Table 2, is at the expense of an OOBE increase of $5 \mathrm{~dB}$. Similar results have been shown in [25] and are omitted here.

The PAPRs of various approaches are plotted in Fig. 5. It is shown that the PAPRs of W-OFDM and F-OFDM are almost the same as that of CP-OFDM. However, for SP-OFDM, W-SP-OFDM, and F-SP-OFDM, their PAPR performances degrade compared with that of CP-OFDM and the degradation increases as $R$ increases. This is due to the fact that the precoded elements in $s$ become correlated. As mentioned in Section 2.3, SP-OFDM, F-SPOFDM, and W-SP-OFDM can be easily extended to reduce the PAPR drastically by using (28). As shown in Fig. 5 , at $\operatorname{Pr}\left(\mathrm{PAPR}>\mathrm{PAPR}_{0}\right)=10^{-3}$, the PAPRs of SPOFDM, W-SP-OFDM, and F-SP-OFDM are reduced by more than $3 \mathrm{~dB}$ if PMix-OFDM, F-PMix-OFDM, and W-PMix-OFDM are used respectively. Therefore, concluding from Figs. 4 and 5, the combination of spectral and PAPR precoding in (28) can suppress the OOBE and reduce the PAPR at the same time without adding extra complexity (compared with spectral precoding in (24)).

\subsection{BER}

In an ideal situation where there is no ACI, FO, or TO, Fig. 6 shows the raw BER versus the $E_{b} / N_{0}$ (the energy per bit to noise PSD ratio) for various approaches. The transmitter is transmitting on subcarriers [-144:-1, 1:156] where QPSK (or 4QAM) modulation is used. The system parameters defined in Tables 1 and $2(N=300)$, and a multipath channel with delay spread of 39 samples are used in the simulation. It is assumed that the channel is perfectly known and a 1-tap MMSE FDE is used at the receiver. Each BER point is obtained by averaging over 10,000 subframes.

In Fig. 6, the BERs of F-OFDMs and W-OFDMs are slightly worse than that of CP-OFDM (denoted as None; representing the case where there is no OOBE suppression process at the transmitter and no ACI rejection at the receiver), due to ISI caused by delay spread. The BER performance degradation mainly depends on the filter or windowing roll-off length, i.e., the F/W-OFDM with longer filter or windowing roll-off length has larger ISI and therefore worse BER performance. On the contrary, all approaches using precoding have small but noticeable BER performance improvements over CP-OFDM due to frequency diversity gain, since each data symbol is modulated by all the subcarriers due to the precoding.

It is remarkable that, with the same amounts of spectral coding rate, the performances of approaches with PMix are much better than approaches with SP. For example, PMix-OFDM, F-PMix-OFDM, and W-PMix-OFDM are much better than those of SP-OFDM, F-SP-OFDM, and W-SP-OFDM (the improvement is larger than $8 \mathrm{~dB}$ at $\mathrm{BER}=10^{-3}$ ) due to the higher diversity gains provided by PMix. This is demonstrated in Fig. 7 which shows the PSDs of several arbitrarily selected modulated data symbols in SP-OFDM and PMix-OFDM. We can see that, in PMix-OFDM, each data symbol $d_{l, i}$ is modulated by all

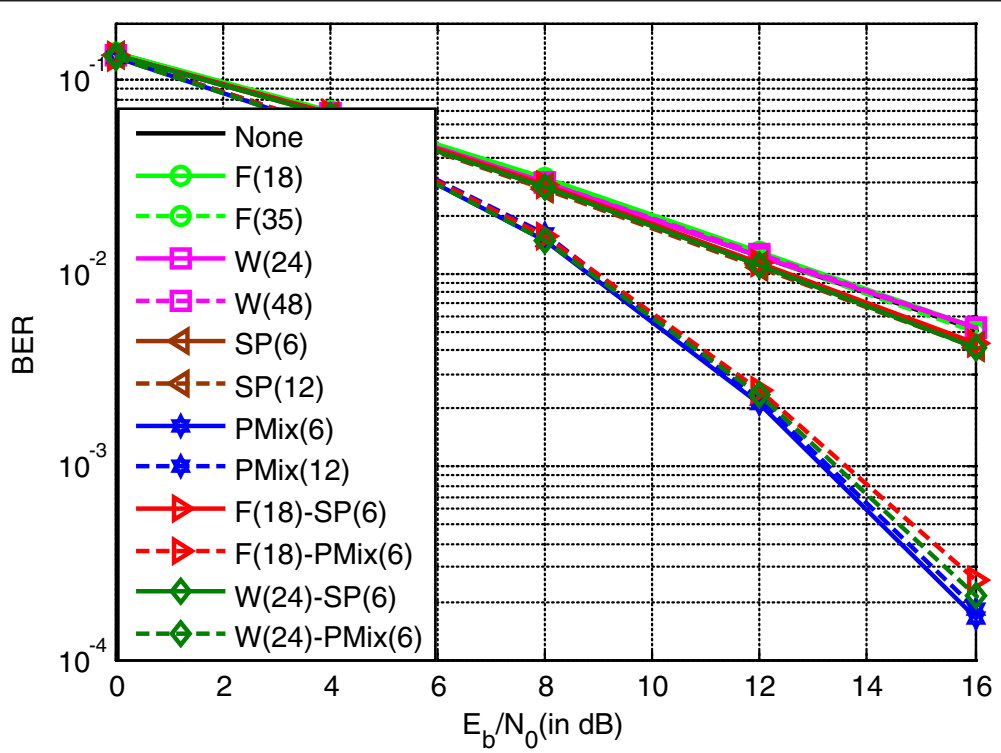

Fig. 6 BER performances when there is no ACl. The meanings of the legends are the same as those in Fig. 4 

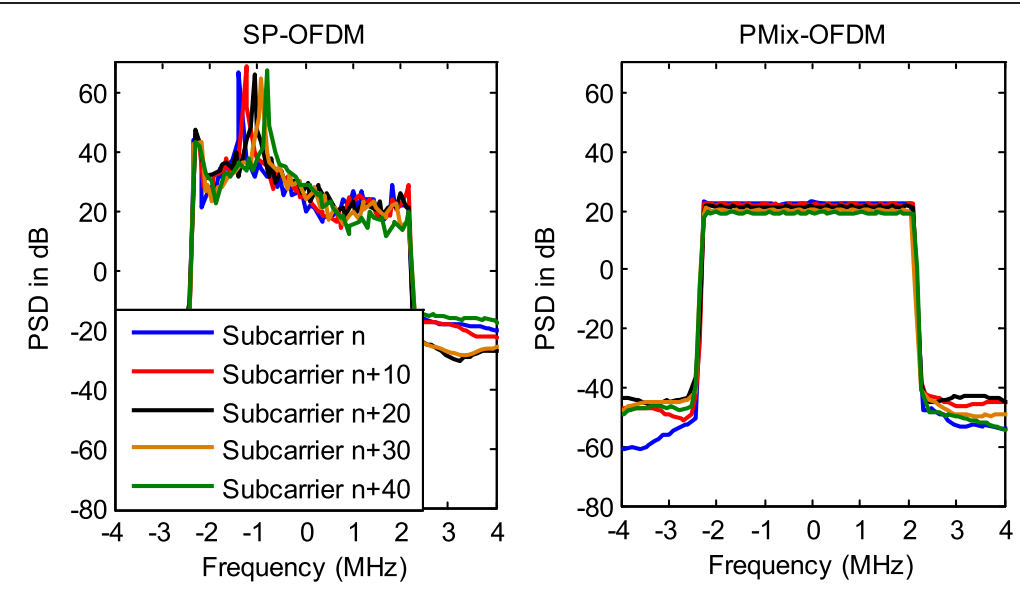

Fig. 7 PSDs of $\sum_{n=0}^{N-1} G_{n, i} d_{l, i} P_{n}(t-I T), i=k, k+10, k+20, k+30, k+40$ in SP-OFDM $\left(\boldsymbol{G}=\boldsymbol{G}_{S}\right)$ and PMix-OFDM $\left(\boldsymbol{G}=\boldsymbol{G}_{S} \boldsymbol{G}_{D F T}\right)$

the subcarriers with almost equal power. However, there is frequency selectivity in SP-OFDM, where the power of each data symbol is spread mainly only on several subcarriers. Thus, in the following simulations where ACI is present, approaches with SP (spectral precoding only) will not be shown since PMix (combined spectral and PAPR precoding) has better BER performance when SP and PMix have the same redundancy length (note that the redundancy length for PAPR precoding is zero).

\section{Practical issues and numerical examples: with $\mathrm{ACl}$}

Various practical issues are addressed in this section and the previous section (Section 3) based on a LTE-like system. Specifically, results shown in this section are with $\mathrm{ACI}$, and results shown in Section 3 are without ACI. The simulation parameters are specified in Table 1, if not otherwise indicated. To examine the effects of ACI on a given receiver, consider two transmitters: one transmitter is desired and generates the desired signal, and the other transmitter is not desired and generates ACI. Presented in this section are the following three scenarios. In the first scenario, the reference receiver belongs to a PU, the desired transmitter belongs to another PU, and the interfering transmitter belongs to a SU. In the second scenario, the reference receiver belongs to a $\mathrm{SU}$, the desired transmitter belongs to another SU, and the interfering transmitter belongs to a PU. In the third scenario, the reference receiver belongs to a $\mathrm{SU}$, the desired transmitter belongs to another $\mathrm{SU}$, and the interfering transmitter belongs to a third SU.

In our simulations, CP-OFDM is used in all PU and SU transmitter-receiver pairs. Note that a PU transmitter does not need to suppress its OOBE to the SU receivers and a PU receiver does not need to have special design to reject ACI from interfering SUs. However, a SU transmitter needs to suppress its OOBE to the PU or non-designated $S U$ receiver, and a $S U$ receiver needs to have special design to reject ACI from the interfering PU and SU transmitters. Thus, we assume no transmit filtering, windowing, or precoding for suppressing OOBE in the PU transmitter. We also assume that in the PU receiver, there is a standard low-pass filter with the cutoff frequency of $4.5 \mathrm{MHz}$ and the filter length of 35 samples (note that the minimum CP length is 72 samples and the channel impulse response length is 39 samples). For the SU transmitters and receivers, any of the approaches tested in Section 3 can be employed. For simplicity, we assume that the SU transmitters and receivers use the same filtering, windowing, precoding, or hybrid approach.

\subsection{OOBE suppression by a SU transmitter}

Consider an example where a transmit-receive pair of PUs are operating in the frequency band with subcarriers [-144:-1, 1:156] and a transmit-receive pair of SUs are operating in the adjacent frequency band of the same bandwidth. The frequency separation of the two bands is 1.5 times of subcarrier spacing (i.e., $22.5 \mathrm{kHz}$ ) where the 0.5 (in 1.5) implies that there is FO between the PU pair and SU pair. Two cases are investigated. In the first case, $\mathrm{TO}=0$, where the PU pair and SU pair are perfectly synchronous in time. In the second case, $\mathrm{TO}=200 / 1024 \mathrm{~T}_{\mathrm{s}}$, where there is a 200-sample delay between the PU pair and SU pair.

The OOBE suppression performance of W-OFDM, FOFDM, PMix-OFDM, F-PMix-OFDM, and W-PMixOFDM by the SU transmitter will be investigated by evaluating the raw BER performance of the PU receiver. In this simulation, the power of the $\mathrm{SU}$ transmitter is $10 \mathrm{~dB}$ higher than that of the PU transmitter. Figure 8 shows the BER performance of the $\mathrm{PU}$ receiver for $\mathrm{TO}=0$ and $\mathrm{TO}=200$ when the $\mathrm{SU}$ transmitter uses various 

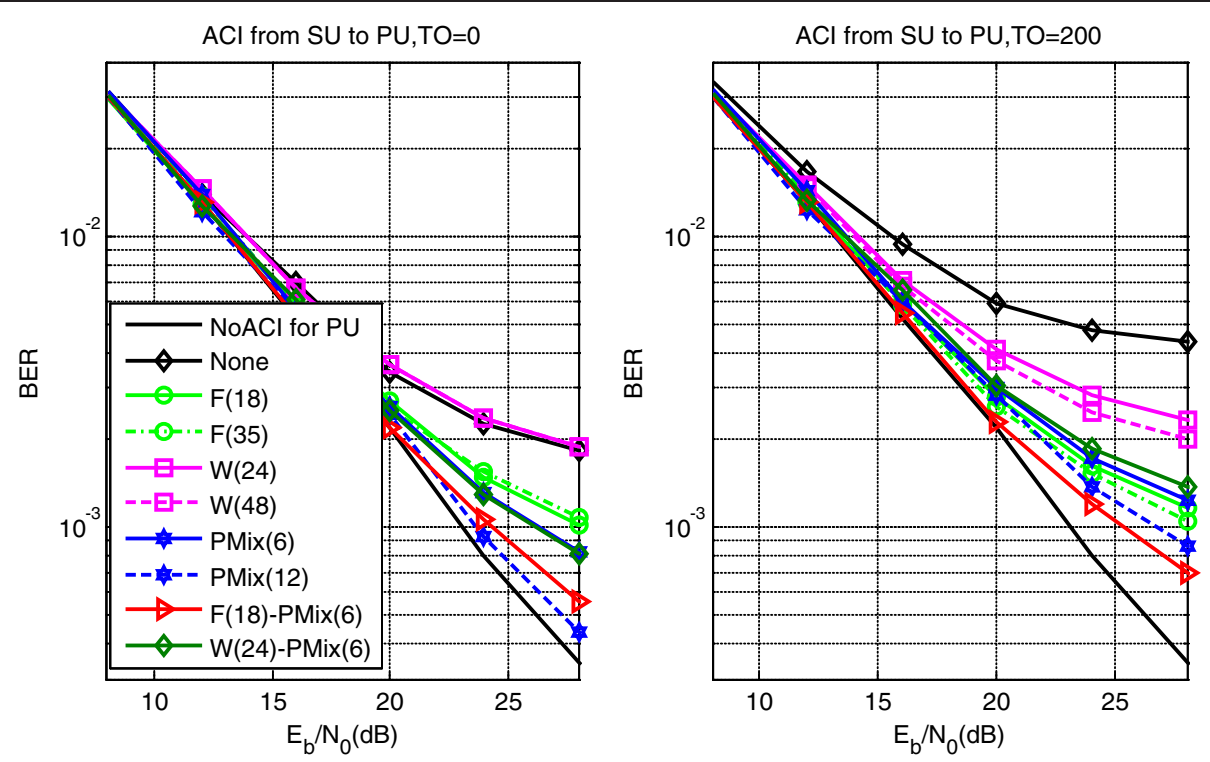

Fig. 8 BER performance of PU accounting for OOBE suppression of a SU transmitter. The meanings of the legends are the same as those in Fig. 4

OOBE suppression approaches. For comparison, the best possible performance (denoted as "No ACI for PU"; representing the case where the interfering SU pair is absent) and the worst possible performance (denoted as None; representing the case where there is no OOBE suppression process in the interfering $\mathrm{SU}$ transmitter) are included in Fig. 8. It is clear that this is an interferencelimited system because error floors exist for all approaches at high signal to noise ratios (SNRs).

When $\mathrm{TO}=0$, Fig. 8 shows that W-OFDM has almost the same OOBE suppression performance as None (i.e., windowing does very little in suppressing OOBE in this example where the frequency spacing of the desired and interfering signals is $22.5 \mathrm{kHz}$ ). This is because WOFDM has larger stopband attenuation but a slower fall-off rate than None (see Fig. 4). For F-OFDM, we observe that the F-OFDM with longer filter length has a slightly worse BER performance, which seems contrary to the spectral containment result in Fig. 4. In fact, there is no contradiction because the BER performances are affected not only by OOBE but also ISI. A filter with longer filter length has smaller OOBE but causes larger ISI. So, the ISI effects dominate the ACI effects in this example. Comparing F-OFDM with W-OFDM, we observe that F-OFDM outperforms W-OFDM. Comparing F-OFDM and W-OFDM with PMix-OFDM, we observe that PMix-OFDM has the best OOBE suppression results but at the cost of a small spectral efficiency loss and a significant complexity increase. Furthermore, the PMix-OFDM with more redundancy has smaller OOBE and causes less interference to the PU. It is remarkable that F(18)-PMix(6)-OFDM outperforms both $F(18)$ OFDM and PMix(6)-OFDM but at the cost of a small spectral efficiency loss and a significant complexity increase. As W-OFDM did little to suppress OOBE in this example, W(24)-PMix(6)-OFDM has the same BER performance as $\operatorname{PMix}(6)$-OFDM. All these observations are consistent with the spectral containment results in Fig. 4.

When TO between the desired and interfering signals changes from 0 to 200, the BER performances of all approaches degrade in different degrees. As shown in Fig. 8, the degradation of None is profound because the ability to reject interferences of the PU receiver depends very much on correct sampling of each interfering OFDM symbol. The degradation of "PMix" is also quite significant because, to a certain extent, the OOBE suppression ability of the interfering $\mathrm{SU}$ transmitter requires the $\mathrm{PU}$ receiver to have correct samples of each interfering OFDM symbols. Windowing has small degradations because its OOBE suppression ability does not depend a lot on the OFDM symbol structure. However, as shown in $\mathrm{TO}=0$ case, windowing is not a good choice since it did very little to suppress OOBE. Filtering has the least degradation because its OOBE suppression ability depends very little on the OFDM symbol structure. As expected, the hybrid approach F-PMix (or W-PMix) has the combined features of filtering (or windowing) and PMix.

In practice, as PU and SU will generally not be synchronized, filtering is then a good choice for OOBE suppression because of its low complexity and robustness to TO. However, filtering alone is usually not adequate to produce sufficient OOBE suppression because the filter length of F-OFDM is limited by the CP length. In order to satisfy the OOBE suppression requirement and to 
minimize the sensitivity with respect to $\mathrm{TO}$, the combination of filtering with precoding would be necessary if the $\mathrm{CP}$ length is not long enough to allow a sufficient long filtering function. Thus, we conclude from this example that the combination of filtering with precoding would be the most efficient way to suppress OOBE of OFDM signal but at the cost of a small spectral efficiency loss and a significant complexity increase. This can be seen in Fig. 8 where, among all results shown, the hybrid approach F(18)-PMix(6)-OFDM for the SU has the best OOBE suppression performance when $\mathrm{TO}=200$.

\section{2 $\mathrm{ACl}$ rejection by a SU receiver}

Consider the same example as in the previous subsection (Section 4.1) where a transmit-receive pair of PUs are operating in the frequency band with subcarriers [-144:-1, 1:156] and a transmit-receive pair of SUs are operating in the adjacent frequency band of the same bandwidth. The frequency separation of the two bands is 1.5 times of subcarrier spacing where the 0.5 (in 1.5) implies that there is FO between the PU pair and SU pair. Two TO cases are investigated. In the first case, $\mathrm{TO}=0$, where the PU pair and SU pair are perfectly synchronous in time. In the second case, $\mathrm{TO}=200 / 1024 \mathrm{~T}_{\mathrm{s}}$, where there is a 200-sample delay between the PU pair and SU pair.

The performances of W-OFDM, F-OFDM, PMixOFDM, F-PMix-OFDM, and W-PMix-OFDM used by the SU receiver to reject the ACI from the PU transmitter will be investigated by evaluating the BER performance of the SU receiver. For comparison, the worst possible performance (denoted as None; representing the case where there is no OOBE suppression or ACI rejection process in the $\mathrm{SU}$ transmitter and receiver) are included in Fig. 9. In this simulation, the power of the PU transmitter is $10 \mathrm{~dB}$ higher than that of the SU transmitter.

When $\mathrm{TO}=0$, the $\mathrm{ACI}$ rejection performance of $\mathrm{F}$ OFDM/W-OFDM mainly depends on the filtering/windowing length (the longer the filter length/windowing roll-off length, the better the ACI rejection performance), and the performance of PMix-OFDM depends on the redundancy length (the longer the redundancy length, the better the ACI rejection performance). In addition, PMix outperforms F-OFDM and W-OFDM. These observations are similar to those made for the OOBE suppression results in Fig. 8. However, unlike the OOBE suppression results in Fig. 8, windowing outperforms filtering for ACI rejection in Fig. 9. This is due to the fact that, at the SU receiver, the process of discarding $\mathrm{CP}$ generates discontinuities of the received signal. Since discontinuities usually cause spread in the frequency, the ACI caused by the interfering signal before discarding $\mathrm{CP}$ is smaller than that after discarding $\mathrm{CP}$. Now that filtering is done before discarding CP, the ACI rejection ability of filtering approaches is greatly reduced by $\mathrm{CP}$ removal. On the other hand, windowing process is carried out on the signal portion that goes through the FFT for rejecting $\mathrm{ACI}$, and the $\mathrm{ACI}$ rejection ability of windowing approaches is unaffected by $\mathrm{CP}$ removal because the discarded portion is compensated (see Fig. 3). For hybrid approaches, F(18)-PMix(6)OFDM outperforms both F(18)-OFDM and PMix(6)OFDM but at the cost of a small spectral efficiency loss and a significant complexity increase. Similarly, W(24)PMix(6)-OFDM outperforms both W(24)-OFDM and PMix(6)-OFDM but at the cost of a small spectral efficiency loss and a significant complexity increase. Remarkably, F(18)-PMix(6)-OFDM outperforms W(24)-PMix(6)-OFDM
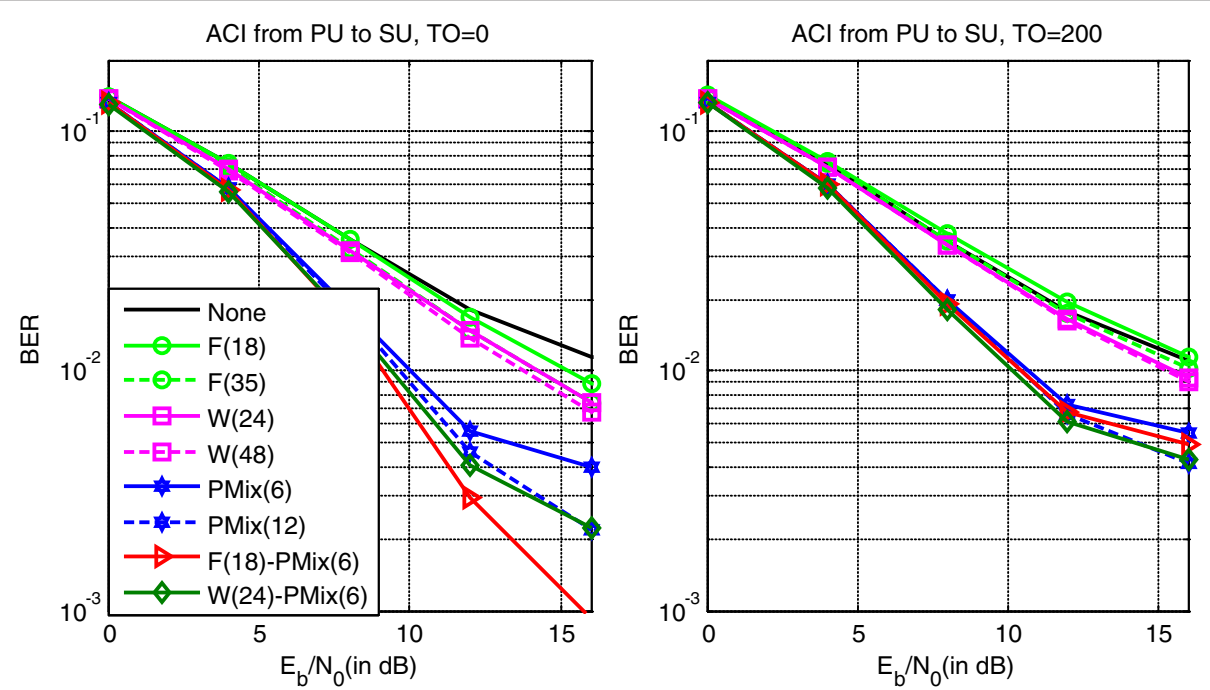

Fig. 9 BER performance of the SU receiver accounting for rejection of the $\mathrm{ACl}$ caused by the PU transmitter. The meanings of the legends are the same as those in Fig. 4 
even though W(24)-OFDM outperforms F(18)-OFDM. It seems that the combination of filtering and spectral precoding work better than the combination of windowing and spectral precoding at $\mathrm{TO}=0$ since both filtering and spectral precoding approaches suppress the unwanted signal in the frequency domain.

When TO changes from 0 to 200, the BER performances of all approaches in Fig. 9 degrade in different degrees. Similar to Fig. 8, the degradation of PMix is significant because, to a certain extent, the ACI rejection ability of the SU receiver requires to have correct samples of each interfering OFDM symbols. Windowing and filtering have smaller degradations because their ACI rejection ability does not depend a lot on the OFDM symbol structure. However, as shown in $\mathrm{TO}=0$ case, filtering is not a good choice since it does not deal with the ACI regeneration due to the CP removal. As expected, the hybrid approach F-PMix (or W-PMix) has the combined features of filtering (or windowing) and PMix. What is remarkably different from the results in Fig. 8 is that BERs for $\mathrm{TO}=0$ and $\mathrm{TO}=200$ are similar for None because $\mathrm{TO}=200$ does not introduce more $\mathrm{ACI}$ than $\mathrm{TO}=0$ when no $\mathrm{ACI}$ suppression technique is employed.

In practice, as PU and SU will generally not be synchronized, windowing is then a good choice for ACI rejection because of its low complexity, robustness to TO, and insensitivity to $\mathrm{CP}$ removal. However, windowing alone is usually not adequate to produce sufficient ACI rejection because the roll-off portion length of the windowing function in W-OFDM is limited by the CP length. In order to satisfy the $\mathrm{ACI}$ rejection requirement and to minimize the sensitivity with respect to $\mathrm{TO}$, the combination of windowing with precoding would be necessary if the CP length is not long enough to allow a sufficient long windowing function. Thus, we conclude from this example that the combination of windowing with precoding would be the most efficient way to reject ACI from interfering OFDM signal but at the cost of a small spectral efficiency loss and a significant complexity increase. This can be seen in Fig. 9 where, among all results shown, the hybrid approach W(24)-PMix(6)-OFDM for the $\mathrm{SU}$ has the best $\mathrm{ACI}$ rejection performance when $\mathrm{TO}=200$.

\subsection{OOBE suppression and $\mathrm{ACl}$ rejection by a $\mathrm{SU}$ (with respect to other SU, with $\mathrm{FO}$ and $\mathrm{TO}$ )}

In this scenario, there are two transmit-receive pairs of SUs operating in adjacent (non-overlapping) frequency bands with the same bandwidth of $4.5 \mathrm{MHz}$. For convenience, we assume that all SUs use the same OOBE/ ACI suppression approach with the parameters defined in Table 2. The frequency separation of the two bands is 1.5 times of subcarrier spacing where the 0.5 implies there is FO between the two pairs. Two cases are investigated. In the first case, $\mathrm{TO}=0$, the two pairs of SUs are perfectly synchronous in time. In the second case, $\mathrm{TO}=200$, there is a 200-sample delay between the two pairs of SUs.

We will evaluate the BER performances of one of the two SU receivers to study the combined effects of OOBE suppression and ACI rejection for various approaches. Among the two SU transmitters, one is chosen to be as desired and the other as unwanted (i.e., an interferer). We assume that the transmit power of the interferer is $10 \mathrm{~dB}$ higher than that of the desired SU transmitter. Any of the approaches discussed in Section 2 can be employed.

Figure 10 shows the BER performances of the desired SU pair with various approaches when $\mathrm{TO}=0$ and 200 .
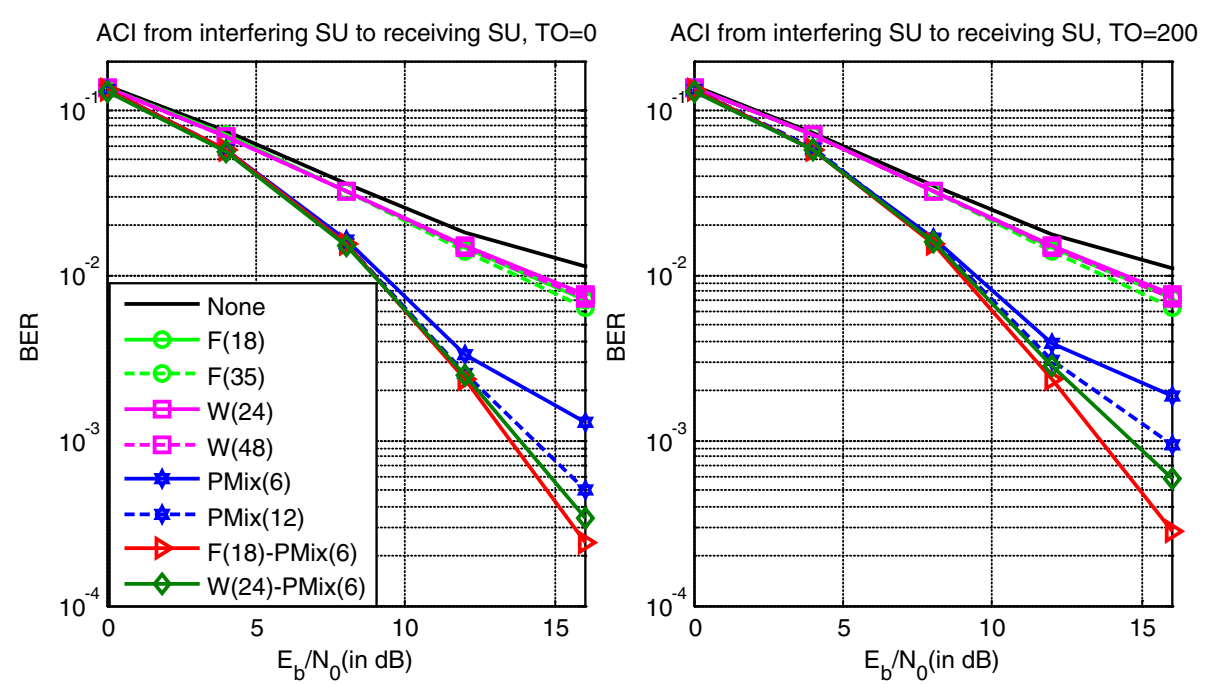

Fig. 10 BER performance of the SU receiver accounting for rejection of the ACl and suppression of OOBE by an interfering SU transmitter. The meanings of the legends are the same as those in Fig. 4 


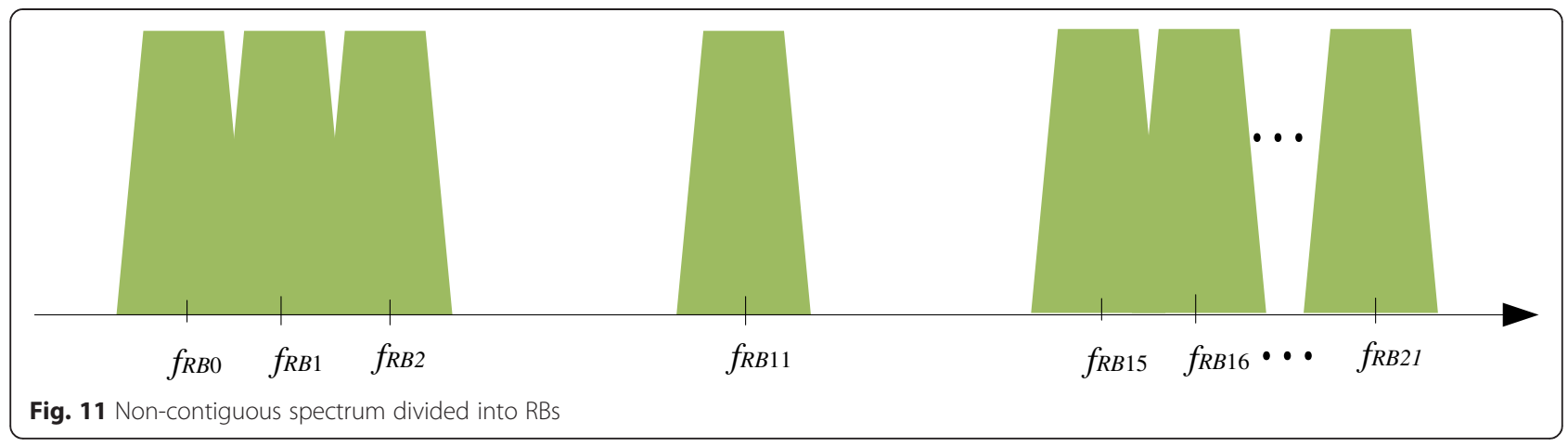

Among all, None is the worst because nothing is done to suppress OOBE or reject ACI; F(18)-PMix(6)-OFDM is the best but at the cost of spectral efficiency loss. For W-OFDM, W(24) and W(48) have the same BER performance. For F-OFDM, that with a longer filter length has a better performance. As we have designed, W(24)-PMix(6)-OFDM and F(18)-PMix(6)-OFDM both achieve better performance than W(24)-OFDM, W(48)OFDM, F(18)-OFDM, F(35)-OFDM, PMix(6)-OFDM, and $\operatorname{PMix}(12)$-OFDM.

When TO is changed from 0 to 200 , there is very little degradation on the performances of None, F-OFDM, and W-OFDM. On the contrary, the degradations on the performance of PMix-OFDM are significant. For the hybrid approaches, the degradations are visible but not significant.

Comparing the BERs of the corresponding approaches in Figs. 9 and 10, the BERs in Fig. 10 (for two pairs of SUs) are smaller than those in Fig. 9 (for one pair of PU and one pair of SUs). This is due to the fact that the transmit interferer in Fig. 10 (i.e., a SU) performs OOBE suppression but the transmit interferer (i.e., a PU) in Fig. 9 does not perform OOBE suppression.

\section{Conclusions}

If the signaling structures are not known, windowing and filtering are the two primary designing principles for the conventional OOBE suppression and ACI rejection approaches. However, when the signaling structures are known, new designs can be added into the conventional designs. For OFDM cognitive radio systems, precoding approaches can be employed to exploit the OFDM signaling structures before IFFT at the transmitter and after FFT at the receiver. In addition, a lot of non-trivial considerations need to be factored into the design. For example, the removal of $\mathrm{CP}$ at the receiver causes $\mathrm{ACI}$ regeneration if the truncation of interference signal due to the $\mathrm{CP}$ removal process is not properly taken into consideration by the adopted ACI rejection approach. On the other hand, effective OOBE is much larger than the estimated OOBE if there is a timing offset (TO) between PU and SU. Thus, among the two conventional approaches, windowing is more appropriate for ACI rejection since the windowing process is carried out on the portion that goes through the FFT and proper compensation for the discarded portion has been made, and filtering is more appropriate for OOBE suppression since it is less sensitive to TO.

In this paper, both time-domain (before FFT at the receiver and after IFFT at the transmitter) and frequencydomain (after FFT at the receiver and before IFFT at the transmitter) OOBE suppression and ACI rejection approaches for OFDM cognitive radio systems are examined

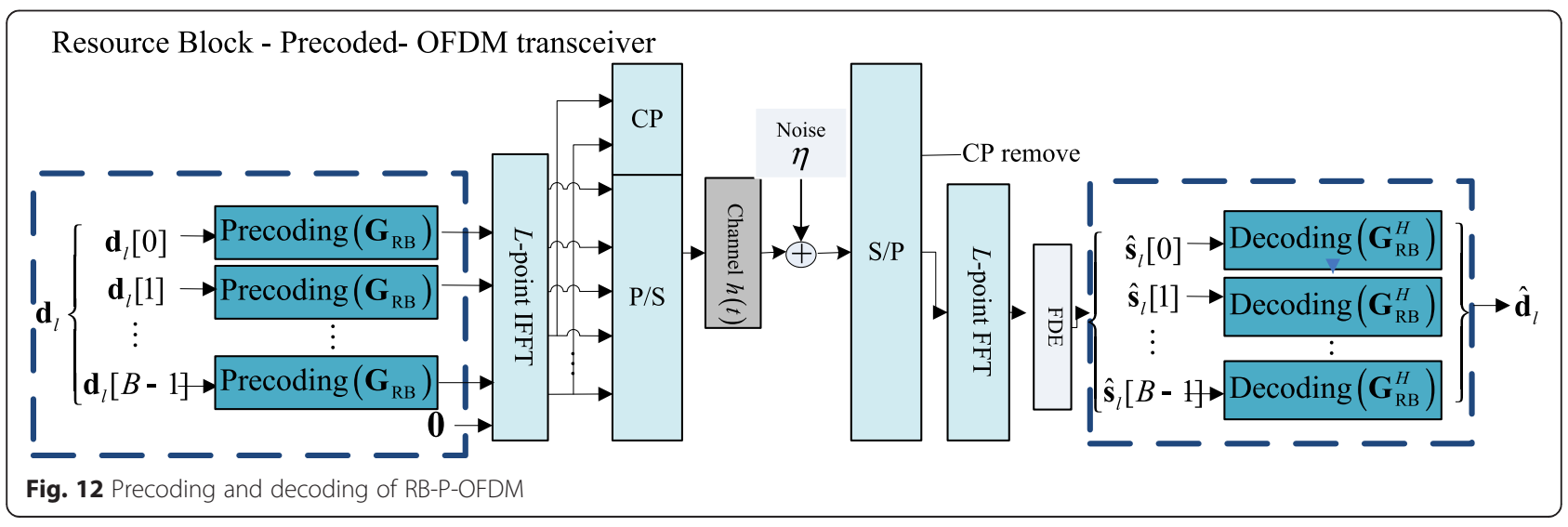


in details. Through the evaluation of the OOBE suppression (a SU transmitter to suppress its OOBE) and ACI rejection (a SU receiver to reject $\mathrm{ACI}$ caused by an interfering $\mathrm{PU}$ or $\mathrm{SU}$ transmitter) performances of all these approaches and their possible combinations under practical conditions (broadband channels with radio impairments such as FO and TO), we see that all of them can be used at the SU transmitter to minimize OOBE or at the SU receiver to reject the ACI. However, each of the existing approaches has some merits and some shortcomings, none of them alone can deal with all practical challenges (such as long multipath delay spread, finite $\mathrm{CP}$ length, requirements of low PAPR, low BER, low complexity and high spectral efficiency, robustness with respect to FO and TO, ability to adapt to non-contiguous and dynamically changing spectrum, etc.) alone. The two timedomain approaches, filtering and windowing, are proved to be efficient approaches to reduce OOBE at the $\mathrm{SU}$ transmitter and reject $\mathrm{ACI}$ at the OFDM-based SU receiver, respectively, because of their robustness with respect to TO. However, their performance will be limited by the $\mathrm{CP}$ length. The developed hybrid precoding approach PMix, which combines spectral precoding and PAPR precoding in the frequency domain, can provide satisfactory performances on OOBE suppression, ACI rejection, PAPR reduction, low BER and robustness with respect to FO, etc., but with the drawbacks of spectral efficiency loss, high complexity (when the available spectrum is broadband, non-contiguous, or time-varying), and being sensitive to TO. Fortunately, the complexity of PMix can be reduced with the resource block scheme introduced in our previous work. Furthermore, to reduce spectral efficiency loss and TO sensitivity of PMix, the idea of combining the frequencydomain PMix with a time-domain approach (filtering or windowing) is presented in details in this paper with simulation results. The combination of PMix with filtering or windowing can mitigate not only the shortcomings of PMix (i.e., high complexity, spectral efficiency loss, and performance loss due to $\mathrm{TO}$ ) but also the shortcomings of filtering and windowing (i.e., sensitivity to delay spread). Simulation results show that F-PMix-OFDM provides the best OOBE suppression and W-PMixOFDM provides the best ACI rejection characteristics in our numerical examples.

\section{Appendix}

\subsection{Resource block scheme}

In a multicarrier modulation system, a RB consists of a fixed number of contiguous subcarriers, which is designated to be the smallest dynamically available transmission band. All the assigned transmission bands or opportunistically detected frequency bands are consisting of one or multiple RBs. As depicted in Fig. 11, a non-contiguous spectrum may be divided into RBs of the same size. Based on the concept of RB, it is possible to remove the dependence of precoding approach on the allocated spectrum.

The main idea of RB-P-OFDM is to precode all RB by a predetermined precoding, $G_{\mathrm{RB}}$, independently. Once $N_{\mathrm{B}}$, the size of each $\mathrm{RB}$, the spacing between the central frequency of each RB, and the notched frequencies, $f_{i}, i=$ $0,1, . ., K-1$, are determined, the matrix $\boldsymbol{P}_{\mathrm{RB}}$ is fixed.

$$
\boldsymbol{P}_{\mathrm{RB}}=\left[\begin{array}{ccc}
P_{0}\left(f_{0}\right) & \cdots & P_{N_{\mathrm{B}}-1}\left(f_{0}\right) \\
\vdots & \ddots & \vdots \\
P_{0}\left(f_{K-1}\right) & \cdots & P_{N_{\mathrm{B}}-1}\left(f_{K-1}\right)
\end{array}\right]
$$

Then, a unique precoding matrix $\boldsymbol{G}_{\mathrm{RB}}$ with coding redundancy $R_{\mathrm{B}}$ can be obtained by (24). In addition, since the dimension of $G_{\mathrm{RB}}$ is decreased from $N$ to $N_{\mathrm{B}}$, the computational complexity of the precoding and decoding operations is greatly reduced.

A block diagram of RB-P-OFDM is shown in Fig. 12. At the transmitter, the data of each RB, $\boldsymbol{d}_{l}[k], k \in U$, is precoded by $G_{\mathrm{RB}}$ independently to form a per-RB precoded data $s_{l}[k]$. Then, all the precoded data $s_{l}[k]$ instead of $\boldsymbol{d}_{l}[k]$ are modulated by the assigned subcarriers. At the receiver, the demodulated data of each $\mathrm{RB}, \hat{\boldsymbol{s}}_{l}[k]$, is decoded independently by $\boldsymbol{G}_{\mathrm{RB}}^{H}$ to recover the per-RB transmitted data $\hat{\boldsymbol{d}}_{l}[k]$. The extension of the RB approach to the W-P-OFDM is straightforward. The extension to the F-P-OFDM is a bit involved but can be done by applying the RB approach to the filtering process as well. However, the RB approach may degrade the spectrum containment unless the redundancy order is increased (which increases the spectral efficiency loss) as discussed in Section 3.2.

\section{Competing interests}

The authors declare that they have no competing interests.

Received: 21 January 2015 Accepted: 4 May 2015

Published online: 26 May 2015

\section{References}

1. J Mitola, GQ Maguire Jr, Cognitive radio: making software radios more personal. IEEE Personal Comm. 6(4), 13-18 (1999)

2. TA Weiss, FK Jondral, Spectrum pooling: an innovative strategy for the enhancement of spectrum efficiency. IEEE Comm. Mag. 42(3), 8-14 (2004)

3. F Meucci, O Cabral, FJ Velez, A Mihovska, NR Prasad, Spectrum aggregation with multi-band user allocation over two frequency bands, in Proc. IEEE Mobile WiMAX Symp, 2009, pp. 81-86

4. Y Wu, WY Zou, Orthogonal frequency division multiplexing: a multi-carrier modulation scheme. IEEE Trans. Consum. Electron. 41(3), 392-399 (1995)

5. S Alamouti, A simple transmit diversity technique for wireless communications. IEEE J. Sel. Areas Comm. 16(8), 1451-1458 (1998)

6. C Cordeiro, K Challapali, D Birru, N Sai Shankar, IEEE 802.22: an introduction to the first wireless standard based on cognitive radios. J. Commun. 1, 1 (2006)

7. A Sahin, H Arslan, Edge windowing for OFDM based systems. IEEE Commun. Lett. 15(11), 1208-1211 (2011)

8. D. Noguet, M. Gautier, V. Berg, Advances in opportunistic radio technologies for TWWS, EURASIP Journal on Wireless Communications and Networking, Nov. 2011, http://jwcn.eurasipjournals.com/content/2011/1/170 
9. I Cosovic, S Brandes, M Schnell, Subcarrier weighting: a method for sidelobe suppression in OFDM systems. IEEE Commun. Lett. 10(6), 444-446 (2006)

10. S Brandes, I Cosovic, M Schnell, Sidelobe suppression in OFDM systems by insertion of cancellation carriers. IEEE 62nd Vehicular Technology Conference (VTC), 2005, pp. 152-156

11. X Fu, J Wang, SQ Li, Sidelobe suppression for OFDM based cognitive radio systems. 4th International Conference on Communications and Networking in China (ChinaCOM), 2009, pp. 1-5

12. CD Chung, Spectrally precoded OFDM. IEEE Trans. Comm. 54(12), 2173-2185 (2006)

13. J Van de Beek, Sculpting the multicarrier spectrum: a novel projection precoder. IEEE Comm. Lett 13(12), 881-883 (2009)

14. J Li, E Bala, R Yang, Resource block filtered-OFDM for future spectrally agile and power efficient systems. Phys. Commun 11, 36-55 (2014)

15. E Bala, J Li, R Yang, Shaping spectral leakage: a novel low-complexity transceiver architecture for cognitive radio. IEEE Vehicular Magazine 8(3), 38-46 (2013)

16. J Fang, L I-Tai, Precoder designs for jointly suppressing out-of-band emission and peak-to-average power ratio in an orthogonal frequency division multiplexing system. IET Commun. 8(10), 1705-1713 (2014)

17. TS 36.211 Evolved Universal Terrestrial Radio Access (E-UTRA); Physical channels and modulation; Release 9, 2010

18. FCC 12-36, Third Memorandum Opinion and Order in the TV white spaces proceeding, ET Docket Nos. 04-186 and 02-380, April 4, 2012.

19. J Van de Beek, Orthogonal multiplexing in a subspace of frequency welllocalized signals. IEEE Comm. Lett 14(10), 882-884 (2010)

20. SB Slimane, Reducing the peak-to-average power ratio of OFDM signals through precoding. IEEE Trans. Veh. Tech. 56(2), 686-695 (2007)

21. I Baig, V Jeoti, PAPR reduction in OFDM systems: Zadoff-Chu matrix transform based pre/post-coding techniques (Second International Conference on Computational Intelligence, Communication Systems and Networks (CICSyN), 2010), pp. 373-377

22. HG Myung, L Junsung, DJ Goodman, Single carrier FDMA for uplink wireless transmission. IEEE Veh. Technol. Mag. 1, 30-38 (2006)

23. HS Malvar, Signal processing with lapped transforms. 357 pages, Artech House, 1992 ISBN 0890064679

24. D Noguet, M Gautier, V Berg, Advances in opportunistic radio technologies for TWWS. EURASIP J. Wireless Commun. Networking 170, 1-12 (2011)

25. J Fang, Z You, I-T Lu, J Li, R Yang, Resource block based precoding schemes for suppressing out-of-band emission. Military Communications Conference (MILCOM), 2013, pp. 1570-1575

\section{Submit your manuscript to a SpringerOpen ${ }^{\circ}$ journal and benefit from:}

- Convenient online submission

- Rigorous peer review

- Immediate publication on acceptance

- Open access: articles freely available online

- High visibility within the field

- Retaining the copyright to your article

Submit your next manuscript at $>$ springeropen.com 